\title{
Spectinomycin Resistance in rpsE Mutants is Recessive in Streptomyces roseosporus
}

\author{
Xiaowei He, Vivian Miao, Richard H. Baltz
}

Received: November 26, 2004 / Accepted: March 16, 2005

(C) Japan Antibiotics Research Association

\begin{abstract}
Eight spontaneous mutants of Streptomyces roseosporus resistant to spectinomycin (SpcR) were mapped to distinct transversions or deletions in the rpsE gene. Most of the mutations were strongly recessive to the wild type SpcS allele. This suggests that some SpcR alleles of $r p s E$ may be useful in a spectinomycin based counterselection system.
\end{abstract}

Keywords spectinomycin, rpsE, ribosomal protein S5, Streptomyces reseosporus, resistance

Spectinomycin is a bacteriostatic aminocyclitol antibiotic that inhibits protein synthesis. It affects ribosome function by binding to the $30 \mathrm{~S}$ subunit and inhibiting translocation of peptidyl-tRNA from the A-site to the P-site $[1,2]$. Translocation involves local pivoting of the head of the ribosome relative to the body, and is sterically hindered by the presence of the antibiotic [3]. Amino acid substitutions in ribosomal protein S5 [4], the product of the $r p s E$ gene, is associated with resistance to spectinomycin (SpcR). This protein is located on the $30 \mathrm{~S}$ subunit and interacts with $16 \mathrm{~S}$ rRNA and other proteins. Crystallographic studies of the Bacillus stearothermophilus S5 protein showed that it contains an $\mathrm{N}$-terminal dsRNA binding region that interacts with 16S rRNA helix H3 [4, 5].

Early experiments on ribosome morphogenesis in E. coli hinted that sensitivity to spectinomycin might be a dominant phenotype. Studies demonstrating assembly of ribosomes from a pool of subunits showed an excess of SpcS strains when isogenic merodiploids were constructed by introducing an $\mathrm{SpcS}$ allele episomally into a SpcR background [6]. Other merodiploids constructed between $\mathrm{SpcR}$ and SpcS strains were unstably SpcS, and segregated SpcR derivatives more frequently than expected from spontaneous mutation [7]. It is possible that $\mathrm{SpcS}$ alleles could be dominant in a heterozygote through incorporation of sensitive subunits into spcR ribosomes $[6,8]$. In this study, we isolated a collection of spontaneous SpcR rpsE mutants of $S$. roseosporus, complemented them with a cloned rpsE gene and showed that $\mathrm{SpcS}$ is dominant over SpcR.

\section{Cloning of the $S$. roseosporus rpsE gene}

The approach used for cloning the rpsE gene took advantage of the synteny observed in the chromosomes of model actinomycetes $[9,10]$, in this case, the local order of genes around rpsE (Fig. 1A). Primers P949 and P950 (5'CGSGARGCSGGVCTSAAGTTC3' and 5'CTTSAGCTTSGGVAGVCGCATGTG3') targeting rplR and rplO were used to amplify $\left(92^{\circ} \mathrm{C} 15\right.$ seconds, $52^{\circ} \mathrm{C} 15$ seconds, $72^{\circ} \mathrm{C} 45$ seconds, 30 cycles) an intervening region from S. roseosporus UA343 (NRRL 15998). The sequenced PCR product (deposited as Genbank AY772011) was found to include $r p s E$ and $r p m D$, indicating that the $r p l R \cdot r p s E \cdot r p m D \cdot r p l O$ continguity was also conserved in $S$. roseosporus (Fig. 1B). The rpsE gene sequence was GC rich $(69.3 \%)$ and the 200 residue deduced S5 peptide was

V. Miao (Corresponding author), X. He, R. H. Baltz: Cubist Pharmaceuticals, Inc., Lexington, MA 02421, E-mail: vmiao@telus.net 
A

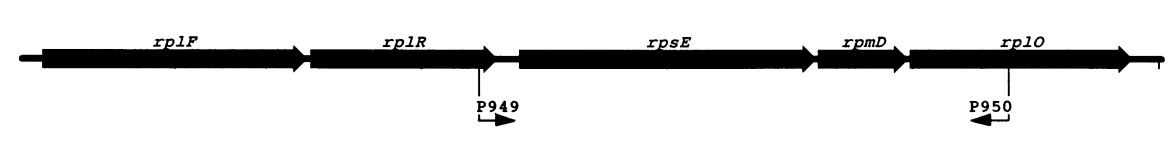

B

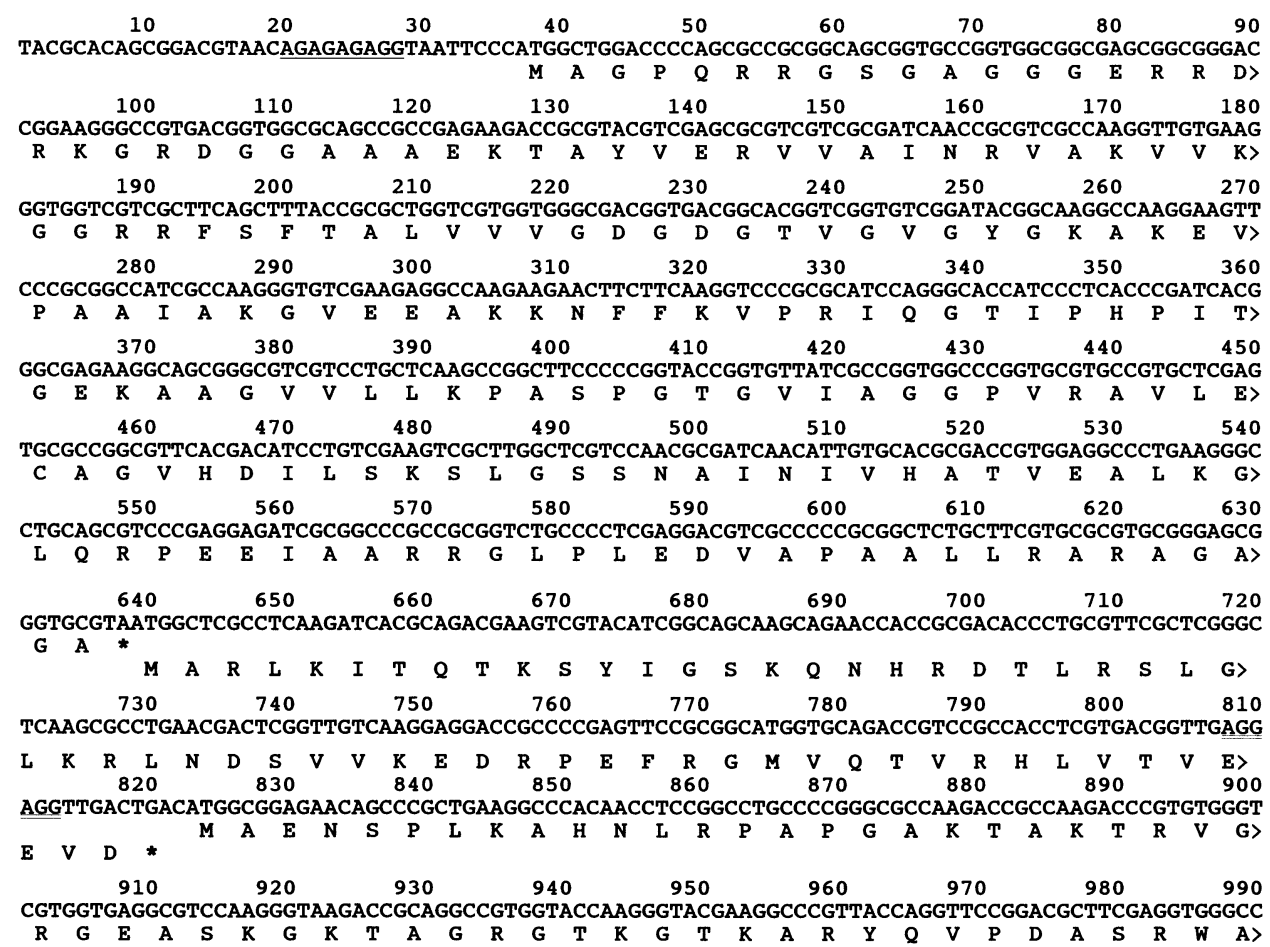

C

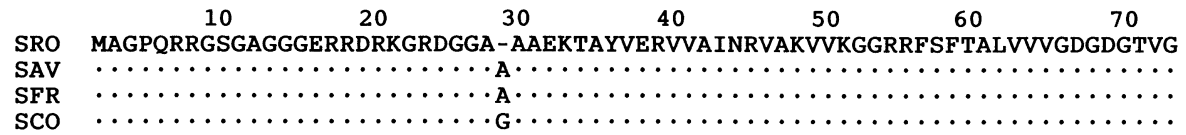

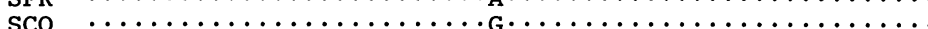

$\begin{array}{lllllll}80 & 90 & 100 & 110 & 120 & 130 & 140\end{array}$

SRO VGYGKAKEVPAAIAKGVEEAKKNFFKVPRIQGTIPHPITGEKAAGVVLLKPASPGTGVIAGGPVRAVLEC

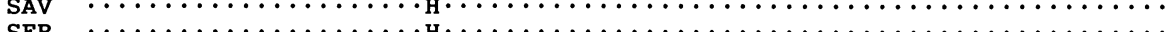

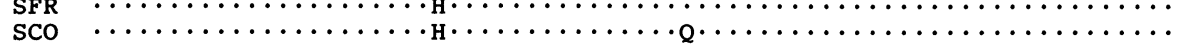

$\begin{array}{llllll}150 & 160 & 170 & 180 & 190 & 200\end{array}$

SRO AGVHDILSKSLGSSNAINIVHATVEALKGLQRPEEIAARRGLPLEDVAPAALLRARAGAGA

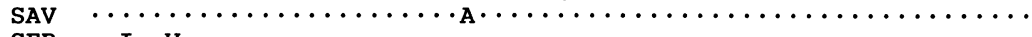

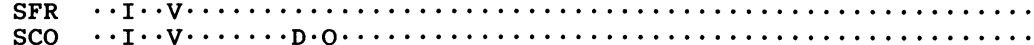

Fig. 1 Organization of rpsE region and characterization of S. roseosporus rpsE and ribosomal protein S5. (A) Organization of rpsE region in Streptomyces coelicolor and location of primers, designed on the basis of $S$. coelicolor data, that were used to amplify the rpsE region of $S$. roseosporus. (B) Sequence and deduced amino acid sequence of $S$. roseosporus rpsE and neighboring genes. A $5^{\prime}$ purine rich sequence (underlined) may serve as a ribosome-binding site (RBS) for rpsE. The coding region extends from nucleotides 37 to 639, and overlaps the coding region for rpmD (639 to 821). The PCR product includes the start of the coding region for $r p / O$ (nucleotide 823) which is preceded by a canonical RBS (double underline). (C) Alignment of S5 from S. roseosporus (SRO), Streptomyces avermitilis (SAV) (Genbank Accession no. NP_826120), S. coelicolor (SCO) (NP_628878) and Streptomyces fradiae (SFR) (AY772012). Dots indicate residues identical in all four streptomycetes. 
Table 1 SpcR mutants and test for dominance

\begin{tabular}{|c|c|c|c|c|c|c|}
\hline \multirow{2}{*}{ Strain ${ }^{a)}$} & \multirow{2}{*}{ Allele } & \multirow{2}{*}{ Mutation in $\mathrm{S}^{\mathrm{b}}$ ) } & \multicolumn{2}{|c|}{$\begin{array}{l}\text { Growth of strain } \\
\text { without pXH006 }\end{array}$} & \multicolumn{2}{|c|}{ 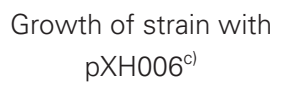 } \\
\hline & & & $\begin{array}{c}0 \mu \mathrm{g} / \mathrm{ml} \\
\mathrm{spc}\end{array}$ & $\begin{array}{c}750 \mu \mathrm{g} / \mathrm{ml} \\
\mathrm{spc}\end{array}$ & $\begin{array}{c}0 \mu \mathrm{g} / \mathrm{ml} \\
\mathrm{spc}\end{array}$ & $\begin{array}{c}750 \mu \mathrm{g} / \mathrm{ml} \\
\mathrm{spc}\end{array}$ \\
\hline UA343 & rpsE & - & + & - & \multicolumn{2}{|c|}{ (not tested) } \\
\hline XH32 & rpsE6 & V43F (GTC > TTC) & + & + & + & - \\
\hline $\mathrm{XH} 31$ & rpsE5 & V43L (GTC >CTC) & + & + & + & - \\
\hline $\mathrm{XH} 27$ & rpsE4 & $\mathrm{A} 44 \mathrm{P}(\mathrm{GCC} \sim>\mathrm{CCC})$ & + & + & + & - \\
\hline $\mathrm{XH} 26$ & rpsE8 & $\Delta$ V46-G49 ( $\Delta 12$ nucleotides) & + & + & + & - \\
\hline $\mathrm{XH} 28$ & rpsE2 & $\mathrm{K} 48 \mathrm{~N}(\mathrm{AAG} \sim>\mathrm{AAC})$ & + & + & + & $(+/-)$ \\
\hline XH33 & rpsE2 & K48N (AAG >AAC) & + & + & \multicolumn{2}{|c|}{ (not tested) } \\
\hline $\mathrm{XH} 29$ & rpsE3 & K48N (AAG > >ATT) & + & + & + & $(+/-)$ \\
\hline $\mathrm{XH} 34$ & rpsE9 & $\Delta$ R51 ( $\Delta 3$ nucleotides) & + & + & \multicolumn{2}{|c|}{ (not tested) } \\
\hline XH35 & rpsE7 & $\mathrm{R} 52 \mathrm{P}(\mathrm{CGC} \sim>\mathrm{CCC})$ & + & + & + & $(+/-)$ \\
\hline $\mathrm{XH} 30$ & $r p s E$ & - & + & + & + & + \\
\hline
\end{tabular}

a) S. roseosporus UA343 is the progenitor strain. Derived strains are listed by the position of mutation. Strains $\mathrm{XH} 26 \sim$ $\mathrm{XH} 30$ were mutants isolated on AS-1 supplemented with $100 \mu \mathrm{g} / \mathrm{ml}$ spectinomycin; the rest were from media with $50 \mu \mathrm{g} / \mathrm{ml}$ spectinomycin. b) Mutations are indicated by the standard one letter amino acid code; e.g. "V43F" indicates that valine at position 43 was changed to phenylalanine, and "(GTC > TTC)" indicates that this was due to a $G$ to $T$ transversion. c) Exconjugants of each strain with pXH006, carrying the wildtype (SpcS) rpsE gene, were patch tested on AS-1 containing spectinomycin and inspected for growth after incubation at $30^{\circ} \mathrm{C}$ for 6 days. " - " indicates no growth, " +" indicates growth comparable to UA343 on media without antibiotic. "+/-" indicates very poor growth after a delay of several days.

extremely similar to homologs found in other streptomycetes (Fig. 1C).

\section{Isolation of SpcR Mutants}

S. roseosporus is sensitive to $<6 \mu \mathrm{g} / \mathrm{ml}$ spectinomycin (J. Penn, personal communication) in AS-1 medium [11]. SpcR colonies were recovered after $3 \sim 15$ days at a frequency of $3.4 \times 10^{-8}$ and $2.7 \times 10^{-8}$ from spores plated on media containing $50 \mu \mathrm{g} / \mathrm{ml}$ or $100 \mu \mathrm{g} / \mathrm{ml}$ spectinomycin, respectively. Ten colonies selected at random were analysed (Table 1). All exhibited wildtype morphology and sporulation, and grew well on AS-1 even with $750 \mu \mathrm{g} / \mathrm{ml}$ spectinomycin. Mutations in $r s p E$, including transversions and deletions, were present in nine of the ten SpcR strains (Table 1). All mutations resided in the ds16S rRNA binding region of S5, "loop 2" as delimited by residues N19 and F33 in the Escherichia coli protein [5, 12], or their equivalent, N41 and F55, in S. roseosporus (Fig. 2). The highly conserved lysines and arginines here form a positively charged $\beta$-ribbon that interacts with the minor groove in 16S rRNA, and the glycine-glycine (GG) residues allow a tight turn before the conserved phenylalanine at the end of the flexible region. As both spectinomycin [3] and "loop 2" interact with 16S rRNA H34 [5], it has been

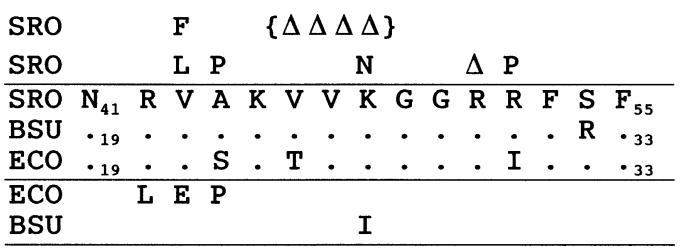

Fig. 2 Deduced mutations in ribosomal protein S5. Residues in the loop 2 region of S5 (delimited by E. coli $\mathrm{N} 19$ and F33) from S. roseosporus (SRO), E. coli (ECO) and $B$. subtilis (BSU) are aligned in the middle of the diagram to show conserved positions. Dots indicate residues identical in all three microorganisms. Individual mutations in SRO are shown at the top, and known ECO and BSU mutations at the bottom. $\Delta$ symbols denote a deletion, or a group of deletions (bracketed).

hypothesized that mutations contributing to greater flexibility in this region may counteract constraints resulting from binding of the antibiotic, thus conferring resistance. Co-localization of the few known SpcR mutations in other bacteria [13 16] to this region is consistent with a mechanism of resistance that involves ribosomal function. The tenth $S$. roseosporus SpcR strain, $\mathrm{XH} 30$, had wildtype rpsE. While it is possible that 
mutations in $16 \mathrm{~S}$ account for non-S5 related SpcR [1, 17], the liklihood is low here because streptomycetes have multiple rRNA operons.

\section{Dominance of SpcS}

To test possible dominance relationships, the coding region of rpsE (nucleotides 40 695, Fig. 1B) was subcloned under control of the strong constitutive $e r m E p^{*}$ promoter in pHM11a, an integrative E. coli/Streptomyces shuttle vector [18]. The resulting plasmid, pXH006, was introduced into E. coli $\mathrm{DH} 10 \mathrm{~B}$ carrying helper plasmid pUZ8002, and transferred by conjugation using standard methods [19] into several SpcR mutants. Two to six exconjugants were patch tested for sensitivity on AS-1 plates with spectinomycin. Growth was completely inhibited at $750 \mu \mathrm{g} / \mathrm{ml}$ spectinomycin in strains carrying rpsE4, rpsE5, rpsE6, or $r p s E 8$, but not in strains with $r p s E 2$, rpsE3, or $r p s E 7$ which showed some growth only after several days (Table 1). Notably, SpcR mutations at the $N$-terminal side of "loop 2" were completely recessive, whereas those at the $C$ terminus (K48-R52) were not. Introduction of pHM11a alone did not affect growth or spectinomycin resistance. That the non-rpsE related resistance in strain $\mathrm{XH} 30$ remained unaffected by the introduction of the wildtype gene supports the specificity of this complementation test.

\section{Utility of rpsE for Counter-selection}

Homologous gene replacement is a useful tool for precise molecular dissection of gene function but has a disadvantage in being laborious. To overcome this, plasmid-borne counter-selection systems based on recessive resistance phenotypes, e.g. rpsL-dependent streptomycin resistance, have been used [20]. The present study suggests that rpsE might be useful in a similar manner. In particular, investigations requiring multiple replacements at one locus may be well served by construction of an intermediate strain in which counterselection, such as provided by $r p s E$, is directly applied to the target locus, rather than to the delivery plasmid. In this arrangement, a dominant allele for sensitivity is placed at the desired target locus and is epistatic over a recessive allele for resistance at the native locus: subsequent homologous recombination of test constructs at the target evicts the dominant allele, and expression of resistance is indicative of successful gene replacement by double crossover. Since $S$. roseosporus rpsE mutations are similar to those in E. coli and B. subtilis, SpcR based counterselection could have a broad applicability. Conservation of $r p s E$ proteins among streptomycetes further suggests that the $S$. roseosporus rpsE gene might confer a dominant sensitivity phenotype when introduced into SpcR strains derived from other streptomycetes.

Acknowledgments The authors thank K. Nguyen for help with conjugation, E. Cundliffe for comments on the manuscript, and J. Davies for useful discussions.

\section{References}

1. Bilgin N, Richter A, Ehrenberg M, Dahlberg AE, Kurland CG. Ribosomal RNA and protein mutants resistant to spectinomycin. EMBO J 9: 735-739 (1990)

2. Burns DJ, Cundliffe E. Bacterial-protein synthesis. A novel system for studying antibiotic action in vivo. Eur J Biochem 37: 570-574 (1973)

3. Carter AP, Clemons WM, Brodersen DE, Morgan-Warren RJ, Wimberly BT, Ramakrishnan V. Functional insights from the structure of the $30 \mathrm{~S}$ ribosomal subunit and its interactions with antibiotics. Nature 407: 340-348 (2000)

4. Bollen A, Davies J, Ozaki M, Mizushima S. Ribosomal protein conferring sensitivity to the antibiotic spectinomycin in Escherichia coli. Science 165: 85-86 (1968)

5. Davies C, Bussiere DE, Golden BL, Porter SJ, Ramakrishnan V, White SW. Ribosomal proteins S5 and L6: high-resolution crystal structures and roles in protein synthesis and antibiotic resistance. J Mol Biol 279: 873-888 (1998)

6. Sparling P, Modolell J, Takeda Y, Davis B. Ribosomes from Escherichia coli merodiplods heterozygous for resistance to streptomycin and to spectinomycin. J Mol Biol 37: 407-421 (1968)

7. Apirion D. Three genes that affect Escherichia coli ribosomes. J Mol Biol 30: 255-275 (1967)

8. Progress in Molecular and Subcellular Biology. SpringerVerlag, Berlin (1969)

9. Ikeda H, Ishikawa J, Hanamoto A, Shinose M, Kikuchi H, Shiba T, Sakaki Y, Hattori M, Omura S. Complete genome sequence and comparative analysis of the industrial microorganism Streptomyces avermitilis. Nat Biotechnol 21: 526-531 (2003)

10. Bentley SD, Chater KF, Cerdeno-Tarraga AM, Challis GL, Thomson NR, James KD, Harris DE, Quail MA, Kieser H, Harper D, Bateman A, Brown S, Chandra G, Chen CW, Collins M, Cronin A, Fraser A, Goble A, Hidalgo J, Hornsby T, Howarth S, Huang CH, Kieser T, Larke L, Murphy L, Oliver K, O'Neil S, Rabbinowitsch E, Rajandream MA, Rutherford K, Rutter S, Seeger K, Saunders D, Sharp S, Squares R, Squares S, Taylor K, Warren T, Wietzorrek A, Woodward J, Barrell BG, Parkhill J, Hopwood DA. Complete genome sequence of the model actinomycete Streptomyces coelicolor A3(2). Nature 417: 141-147 (2002)

11. Baltz RH. Genetic recombination by protoplast fusion in Streptomyces. Dev Indust Microbiol 21: 43-54 (1980)

12. Ramakrishnan V, White SW. The structure of ribosomal protein S5 reveals sites of interaction with 16S rRNA. 
Nature 358: 768-771 (1992)

13. DeWilde M, Wittmann-Liebold B. Localization of the amino-acid exchange in protein S5 from an Escherichia coli mutant resistant to spectinomycin. Mol Gen Genet 127: 273-276 (1973)

14. Funatsu G, Schiltz E, Wittmann HG. Ribosomal proteins. XXVII. Localization of the amino acid exchanges in protein S5 from two Escherichia coli mutants resistant to spectinomycin. Mol Gen Genet 114: 106-111 (1971)

15. Funatsu G, Nierhaus K, Wittmann-Liebold B. Ribosomal proteins. XXII. Studies on the altered protein S5 from a spectinomycin-resistant mutant of Escherichia coli. J Mol Biol 64: 201-209 (1972)

16. Itoh $\mathrm{T}$. Amino acid replacement in the protein $\mathrm{S} 5$ from a spectinomycin resistant mutant of Bacillus subtilis. Mol Gen
Genet 144: 39-42 (1976)

17. Brink MF, Brink G, Verbeet MP, de Boer HA. Spectinomycin interacts specifically with the residues G1064 and C1192 in 16S rRNA, thereby potentially freezing this molecule into an inactive conformation. Nucleic Acids Res 22: 325-331 (1994)

18. Motamedi H, Shafiee A, Cai SJ. Integrative vectors for heterologous gene expression in Streptomyces spp. Gene 160: 25-31 (1995)

19. Kieser T, Bibb MJ, Buttner MJ, Chater KF, Hopwood D. Practical Streptomyces Genetics. John Innes Foundation, Norwich (2000)

20. Hosted TJ, Baltz RH. Use of rpsL for dominance selection and gene replacement in Streptomyces roseosporus. J Bacteriol 179: 180-186 (1997) 